\title{
Loss of Sigma-1 Receptor Chaperone Promotes Astrocytosis and Enhances the Nrf2 Antioxidant Defense
}

\author{
Tzu-Yu Weng, ${ }^{1}$ Denise T. Hung, ${ }^{2}$ Tsung-Ping Su, ${ }^{2}$ and Shang-Yi A. Tsai ${ }^{2}$ \\ ${ }^{1}$ Genomics Research Center, Academia Sinica, Taipei, Taiwan \\ ${ }^{2}$ Cellular Pathobiology Section, Integrative Neuroscience Research Branch, Intramural Research Program, National Institute on Drug \\ Abuse, Department of Health and Human Services, National Institutes of Health, Baltimore, MD 21224, USA
}

Correspondence should be addressed to Shang-Yi A. Tsai; stsai@mail.nih.gov

Received 31 March 2017; Revised 9 June 2017; Accepted 28 June 2017; Published 13 August 2017

Academic Editor: Mark G. Waugh

Copyright @ 2017 Tzu-Yu Weng et al. This is an open access article distributed under the Creative Commons Attribution License, which permits unrestricted use, distribution, and reproduction in any medium, provided the original work is properly cited.

Sigma-1 receptor (Sig-1R) functions as a chaperon that interacts with multiple proteins and lipids and is implicated in neurodegenerative and psychiatric diseases. Here, we used Sig-1R KO mice to examine brain expression profiles of astrocytes and ubiquitinated proteins, which are both hallmarks of central nervous system (CNS) pathologies. Our results showed that Sig-1R KO induces increased glial fibrillary acidic protein (GFAP) expression in primary neuron-glia cultures and in the whole brain of fetus mice with concomitantly increased accumulations of ubiquitinated proteins. Astrogliosis was also observed in the neuron-glia culture. Upon proteasome or autophagy inhibitor treatments, the pronounced ubiquitinated proteins were further increased in Sig-1R KO neurons, indicating that the Sig-1R regulates both protein degradation and quality control systems. We found that Nrf2 (nuclear factor erythroid 2-related factor 2), which functions to overcome the stress condition, was enhanced in the Sig-1R KO systems especially when cells were under stressful conditions. Mutation or deficiency of Sig-1Rs has been observed in neurodegenerative models. Our study identifies the critical roles of Sig-1R in CNS homeostasis and supports the idea that functional complementation pathways are triggered in the Sig-1R KO pathology.

\section{Introduction}

Sigma-1 receptor (Sig-1R) is a transmembrane chaperone protein that resides in the endoplasmic reticulum (ER). Enriched in the mitochondrion-associated ER membrane (MAM) domain where there are ample cholesterol and lipids [1], the Sig-1R has been implicated in many neurodegenerative and psychiatric diseases, as well as in drug-abuseregulated synaptic plasticity [2-4]. As a chaperone protein, the Sig-1R interacts with ion channels, receptors [5], and lipids [6] and is noted for its interaction with another ubiquitously expressed chaperone, $\mathrm{BiP}$ (also known as GRP78), during the resting state. Upon stress induction or ligand stimulation, the Sig-1R disassociates from BiP and translocates to other cellular compartments to regulate ion channels or other receptor activities, such as inositol 1,4,5-trisphosphate type 3 (IP3) receptor activity [3,7]. Sig-1Rs support cellular survival by maintaining mitochondrial homeostasis, $\mathrm{Ca}^{2+}$ concentrations [8], and ER stress responses [9]. The
Sig-1R can translocate to the plasma membrane proximity upon ligand stimulation, modulates cyclin-dependent kinase 5 (cdk5) activity via lipid modifications [10], or translocates to the nuclear membrane to regulate gene transcription [11]. The Sig-1R plays promising roles not only in neuroprotection but also against other diseases [12]. Sig-1Rs are expressed higher in cancer tissues and correlated to decreased patient survival $[13,14]$ by influencing $\mathrm{Ca}^{2+}$ homeostasis and are participating in the apoptosis-induced caspase activation [15]. Sig-1R antagonists also show combined effects against cancers through ER stress and reactive oxygen species (ROS) as well as the induction of the apoptotic pathways [16]. In addition, Sig-1Rs function in lipid transport and regulations [6, 17], autophagy [18, 19], and inflammatory responses $[3,20]$.

The Sig-1R exists abundantly both in neurons and glia, implying that Sig-1Rs are involved in multiple physiological and pathological processes. Several lines of evidence support the hypothesis that the Sig-1R plays an important role in 
neuroprotection $[21,22]$. Silencing of Sig-1Rs in primary neurons resulted in decreased mitochondrial membrane potential and aberrant formation of mitochondrial aggregates [10,23], reduced spine formation, enhanced superoxide productions, hampered axonal extension [10, 23], reduced axonal density, and induced accumulation of phosphorylated tau and phosphorylated neurofilaments [10]. These phenomena are closely related to the stress conditions that characterize certain neurodegenerative diseases. Sig-1Rs have been linked to certain patients with neurological disorders. Namely, mutations of the Sig-1R were found in amyotrophic lateral sclerosis (ALS) cohorts [24, 25], and reduced density of Sig-1Rs was observed in the cerebral and cerebellar regions of the brain in early Alzheimer's disease (AD) patients [26]. In animal models, Sig-1R KO mice exhibit the phenotypes of motor neuron degeneration, reduced ER-mitochondrial contacts, and perturbation of mitochondrial and calcium homeostasis [27]; depletion of Sig-1R in the SOD1*G93A or SOD $1^{*}$ G85R mouse also accelerates ALS progression and is accompanied by MAM disruption [25, 28]. In addition, the Sig-1R agonist PRE-084 can alleviate the pathological and behavioral defects in experimental models of Parkinson's disease (PD) and ALS via upregulating neurotrophic factors or restoring Sig-1R's functions [25, 29]. Here, we sought to investigate hallmarks of neurodegeneration in the Sig-1R KO brain.

Astrocytes are functionally diverse in the central nervous system (CNS) and are gaining recognition in the pathological processes of neurodevelopmental and neurodegenerative diseases [30]. Emerging evidence indicates that astrocytes not only serve as supporting cells to maintain neuronal activity and survival, but are also involved in maintaining the integrity of the CNS. Upon brain insults, astrocytes are recruited to the injured sites where they may provide neuroprotective effects [31]. The double-edged sword role of astrocyte activation and overactivated gliosis accompanies many CNS pathologies [32]. In neurodegenerative diseases, reactive gliosis or astrocytosis [32] and enhanced glial fibrillary acidic protein (GFAP) expression are occurring in concert with neuronal degeneration [33]. Inhibition of the Sig-1R reduced the pathological activation of astrocytes in mechanical allodynia [34]. In the striatum, Sig-1Rs can activate astrocytes under methamphetamine treatment conditions via a selfactivation mechanism [35], suggesting a complex yet important role of Sig-1R in balancing astrocyte activity in brain hemostasis. Sig-1Rs also regulate the oxidative stress system in the retinal glia cells via the Nrf2- (nuclear factor erythroid 2-related factor 2-) dependent system [36].

Production of excess ROS or imbalance of the antioxidative system is closely related to neurodegenerative disease [37]. A previous report found that the antioxidant protein Prdx6 was increased in the liver of Sig-1R KO animals compared to that of the WT mice [38]. Microarray data also revealed that silencing of Sig-1Rs in primary hippocampal neurons induced expression of genes related to the Nrf2mediated oxidative stress pathway [39]. The Nrf2 is a transcription factor that binds to the antioxidant response element (ARE) and controls genes that participate in cellular defense against oxidative insults $[40,41]$. Additionally, the
Sig-1R has been shown to be involved in the functional activity of Nrf2 and ARE signaling pathways in retinal glia cells, liver, and lung tissues $[36,38]$. We also examined the Nrf2 expression patterns in CNS-derived primary cultures from our Sig-1R KO system.

We previously reported that knocking down of Sig-1R increased the total amount of ubiquitinated proteins in neuronal cultures [10]. Sig-1R KO mice also display shorter axons and diminished axonal density compared to control mice [10]. To address the role of Sig-1Rs in neurodegeneration, we investigated the phenotype of Sig-1R KO mice. We hypothesize that the level of Sig-1Rs is critical in neurodegeneration by either invoking the activation of astrocytes or Nrf2. Characterization of the CNS or primary cultures from Sig-1R KO mice showed astrogliosis in addition to accumulation of ubiquitinated proteins, which may contribute to Sig-1R-deficiency-related pathology. We also observed an enhanced Nrf2 expression in Sig-1R KO HEK cells, neuronal-glial cultures, and astrocytes. This study supports the idea that functional complementation of opposing signaling pathways concerning cellular survival is triggered in Sig-1R KO mice.

\section{Materials and Methods}

2.1. Sig-1R Knockout Mice. Sig-1R KO mice were generated as previously described [10]. We obtained the mice of Oprs1 mutant (+/-) OprsGt(IRESBetageo)33Lex litters on a C57BL/6J $\times 129 s / S v E v$ mixed background from the Mutant Mouse Regional Resource Center of the University of California, Davis. The Sig-1R (+/-) males were backcrossed for 10 generations to females on $\mathrm{C} 57 \mathrm{BL} / 6 \mathrm{~J}$ and then, mice were further genotyped. Mice were maintained in a 12-hour day/night cycle facility with free access to food and water. All animal studies adhered to protocols approved by the National Institutes of Health and the National Institute on Drug Abuse Intramural Research Program Animal Care and Use Program, which is accredited by the Association for Assessment and Accreditation of Laboratory Animal Care International.

2.2. Cells and Treatments. Primary cortical neurons were isolated as previously described [23]. Briefly, E18 fetus cortical neurons were dissected and incubated in $0.05 \%$ trypsinEDTA for $15 \mathrm{~min}$ followed by trypsin inhibitor neutralization. After centrifugation, the supernatant was removed and the cell pellet was resuspended in a Neurobasal medium supplemented with B27, penicillin/streptomycin, and GlutaMAX-I (Thermo Scientific). A Pasteur pipette was used to dissociate the cells, and then, a cell strainer was used to separate the cells from tissue debris. Neuronal cultures were plated on a dish coated with poly-D-lysine hydrobromide and incubated in a $37^{\circ} \mathrm{C}, 5 \% \mathrm{CO}_{2}$ incubator. B27 supplement without the antioxidants (AO) (Thermo Scientific) was used in this study to induce oxidative stress. Astrocytes were isolated according to the modified procedure [42]. Briefly, the isolated brain from 1-day-old neonatal mice was minced by a razor and incubated in $0.25 \%$ trypsin for $10 \mathrm{~min}$ with occasional shaking every $2 \mathrm{~min}$. DNase was added prior to neutralization trypsin using fetal bovine serum (FBS). After 
centrifugation, the supernatant was removed and the cell pellet was resuspended in DMEM containing 10\% FBS and penicillin/streptomycin followed by filtration with a cell strainer. Cells were plated on flasks coated with poly-D-lysine hydrobromide and incubated in a $37^{\circ} \mathrm{C}, 5 \% \mathrm{CO}_{2}$ incubator. After 10 days, cells were vigorously shaken and washed to remove microglia cells. Remaining cells were trypsinized and transferred to new flasks. After 7 days, the flasks were vigorously shaken and washed, and the cells were trypsinized and transferred to plates coated with poly-D-lysine hydrobromide for further assays. CHO, Neuro-2a, or HEK cells were cultured in MEM or DMEM containing 10\% FBS and penicillin/streptomycin and maintained at a $37^{\circ} \mathrm{C}, 5 \% \mathrm{CO}_{2}$ incubator. PolyJet reagent (SignaGen Laboratories) was used for Sig-1R EYFP or Flag-Nrf2 [43] (Addgene) plasmid DNA transfections. For the reagent treatments, primary cultures or cell lines were treated with $10 \mu \mathrm{M} \mathrm{MG132}$ (Sigma), $1 \mu \mathrm{g} / \mathrm{ml}$ cycloheximide (CHX) (Sigma), and $50 \mu \mathrm{M}$ chloroquine (CQ) (Sigma).

2.3. Western Blotting. Cells were washed with PBS and the lysate was collected on ice by a cell scraper with RIPA buffer ( $50 \mathrm{mM}$ Tris, $150 \mathrm{mM} \mathrm{NaCl}, 0.2 \%$ sodium deoxycholate, $0.1 \%$ SDS, $1 \%$ Triton X-100, and adjusted to $\mathrm{pH} 7.2$ ) supplemented with protease/phosphatase inhibitors and MG132; following collection, the lysates were sonicated and then centrifuged at $14000 \mathrm{rpm}$ for $10 \mathrm{~min}$ at $4^{\circ} \mathrm{C}$. Whole brain tissue was also lysed with the RIPA buffer containing protease/phosphatase inhibitors and MG132 followed by sonication. Brain tissue homogenates were collected after centrifugation at $14000 \mathrm{rpm}$ for $10 \mathrm{~min}$ at $4^{\circ} \mathrm{C}$. Protein concentrations were determined, and then, proteins were separated by SDS-PAGE and transferred to polyvinylidene difluoride membranes. Membranes were blocked with 5\% nonfat milk for $1 \mathrm{~h}$ at room temperature and then incubated with the primary antibody overnight at $4^{\circ} \mathrm{C}$. Membranes were then washed and incubated with the species-specific Alexa fluorescence secondary antibody (Thermo Scientific). Signals were acquired using the Kodak Image Station 4000 MM (Kodak) and analyzed by the Carestream MI or ImageJ software. Antibodies employed included the following: polyclonal anti-Sig-1R antibody produced by ourselves $(1: 1000)$, anti-GFAP antibody (number: ab2760, 1:5000) (Abcam), anti-ubiquitin antibody (number: 04-263, 1:1000) (Millipore), anti-Nrf2 antibody (number: 12721, $1: 1000$ ) (Cell signaling), anti-Flag antibody (number: F1804, 1:1000) (Sigma), anti- $\beta$ actin antibody (number: A5441, 1:5000) (Sigma), anti-actin antibody (sc-1616, $1: 1000$ ) (Santa Cruz), anti-ERK antibody (number: sc-271270, 1:1000) (Santa Cruz), anti-tubulin antibody (number: T5168, 1:40000) (Sigma), and anti-Nup62 antibody (number: 610498, $1: 2000$ ) (BD).

2.4. Immunofluorescence. The cells were seeded on 12-well plates or coverslips coated with poly-D-lysine hydrobromide, fixed in $4 \%$ paraformaldehyde, and washed with $0.1 \%$ Triton X-100 in PBS. The fixed cells were blocked with $10 \%$ normal goat serum for $1 \mathrm{~h}$ at room temperature followed by incubation with primary antibodies at $4^{\circ} \mathrm{C}$ overnight. Cells were incubated with Alexa fluorescence secondary antibody
(Thermo Scientific) for $1 \mathrm{~h}$ in the dark. Cells were then washed and kept in the PBS. Coverslips were further mounted with the antifading mounting reagent (Thermo Scientific). Images were taken using the Modular Laser System 2.0 (PerkinElmer) with the Nikon ECLIPSE TE2000-E confocal system (Nikon) or the Zeiss Axiovert $200 \mathrm{M}$ microscope (Zeiss). Antibodies used in this research included the following: anti-GFAP antibody (number: ab2760, 1:5000; number: MAB360, 1:400) (Abcam; Millipore), anti-MAP2 antibody (number: MAB3418, 1:200) (Millipore), anti-LC3 antibody (number: 12741, 1:100) (Cell signaling), anti-p62 antibody (number: PM045, 1:500) (MBL), and anti-Nrf2 antibody (number: 12721, 1:100) (Cell signaling).

2.5. Immunoprecipitation. For the immunoprecipitation assay, cells were washed with ice-cold PBS and then crosslinked with $100 \mu \mathrm{g} / \mathrm{ml}$ DSP in PBS on ice for $20 \mathrm{~min}$. Tris$\mathrm{HCl}(\mathrm{pH} 8.8)$ was added at a final concentration of $50 \mathrm{mM}$ to stop crosslinking for $15 \mathrm{~min}$. Cells were washed and harvested in ice-cold PBS and then centrifuged (3500 rpm) for $15 \mathrm{~min}$ at $4^{\circ} \mathrm{C}$. Cells were lysed in the RPIA buffer containing protease/phosphatase inhibitors and MG132, and the lysate was collected after centrifugation at $14000 \mathrm{rpm}$ for $10 \mathrm{~min}$ at $4^{\circ} \mathrm{C}$. The lysate was incubated overnight with the antibody of interest and then incubated with protein A/G PLUS beads (Santa Cruz) for $1.5 \mathrm{~h}$. Beads were washed with modified RIPA buffer $(50 \mathrm{mM}$ Tris, $150 \mathrm{mM}$ $\mathrm{NaCl}, 0.05 \%$ sodium deoxycholate, $0.05 \%$ SDS, $0.5 \%$ Triton $\mathrm{X}-100$, and adjust to $\mathrm{pH} 7.2$ ) containing protease/phosphatase inhibitors and MG132. Immunoprecipitated samples were preserved in the $2 \times$ Laemmli sample buffer (BioRad) containing 2-mercaptoethanol. Antibodies employed included the following: anti-Nrf2 antibody (number: 12721, $1: 50$ ) (Cell signaling), anti-Flag antibody (number: F1804, $1: 100$ ) (Sigma), normal mouse IgG (number: sc-2025) (Santa Cruz), and normal rabbit IgG (number: sc-2027) (Santa Cruz).

2.6. Nuclear Fractionation Assay. WT and Sig-1R KO HEK cells (generated using the CRISPR KO system) were seeded onto the dish for $80 \%$ confluency overnight and then transfected with Flag-Nrf2 plasmid using PolyJet reagent. DMSO or $10 \mu \mathrm{M}$ MG132 was treated for $24 \mathrm{~h}$ after transfection. After $24 \mathrm{~h}$ of MG132 treatment (48 h posttransfection), nuclear fractions were collected with the Subcellular Protein Fractionation Kit for Cultured Cells (number: 78840, Thermo Scientific). Reagents were supplemented with the protease inhibitor and MG132, and the nuclear protein fractions were collected sequentially using the buffer provided according to the manual. Protein concentrations were determined and then subject to Western blotting.

\section{Results}

3.1. Mixed Neuronal-Glial Cultures Derived from Sig-1R KO Animals Are Prone to Astrocyte Activation. To test whether gliosis occurs in Sig-1R KO mice, we examined expression of GFAP in the animal brains as well as in the primary mixed neuronal-glial cultures. Whole brain homogenates from E18 


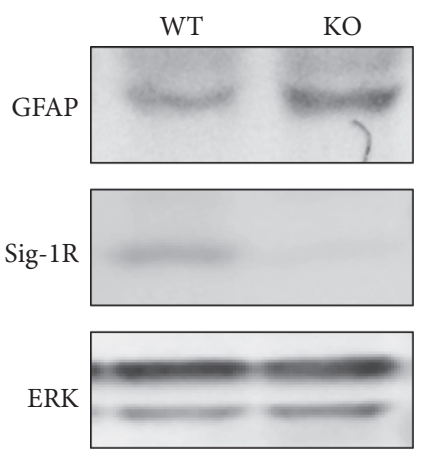

(a)

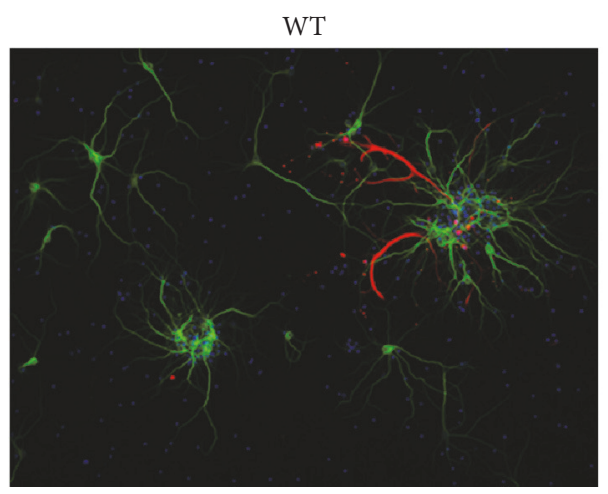

WT

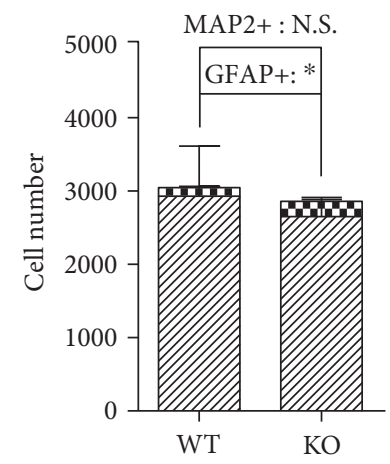

WII MAP2+

B GFAP+

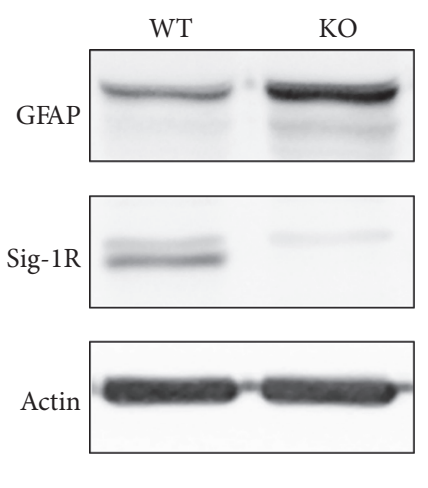

(b)

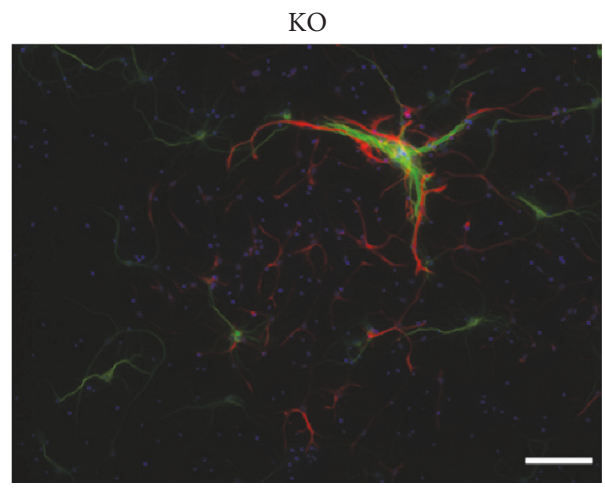

Scale bar $=100 \mu \mathrm{m}$

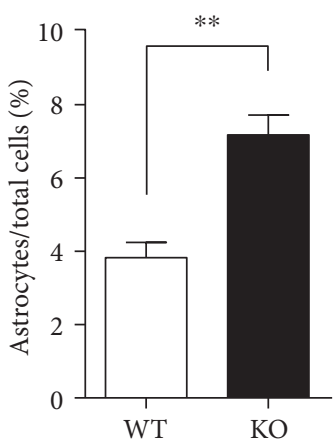

FIgURE 1: Pronounced astrocyte populations are observed in Sig-1R KO mixed neuronal-glial cultures. The whole brains of (a) E18 fetuses $(N=3)$ from Sig-1R WT or KO mice were collected and homogenized for GFAP immunoblotting. (b) Primary neuronal-glial cultures were isolated from Sig-1R WT or KO E18 fetuses. Cell lysates were harvested on DIV 11 for GFAP immunoblotting. Deficiency of the Sig-1R induced more GFAP expression in the fetus as well as in the primary neuronal-glial cultures. (c) Isolated mixed neuronalglial cultures from Sig-1R WT or KO E18 fetuses were seeded at a density of $5 \times 10^{4}$ cells per well and stained with the neuron and astrocyte markers MAP2 (green) and GFAP (red), respectively, on DIV 11. Sig-1R KO mixed neuronal-glial cultures have more GFAPpositive astrocytes. In the graph, GFAP was normalized to the internal control. The GFAP expression level in the WT was normalized to 1. Error bar indicates SEM. ${ }^{*} p<0.05,{ }^{* *} p<0.01, t$-test.

Sig-1R KO fetuses showed increased GFAP expression (Figure 1(a)). Enhanced GFAP expression was also observed in the Sig-1R KO mixed cultures compared to that of WT on DIV 11 (Figure 1(b)), suggesting a pronounced astrocyte population and increased astrocytic activities in the $\mathrm{KO}$ neuronal-glial cultures. Immunofluorescence data also revealed that Sig-1R KO neuronal-glial cultures exhibited increased numbers of GFAP-positive astrocytes and an increased GFAP ratio compared to the WT cells as both cultures were maintained under the same culture 


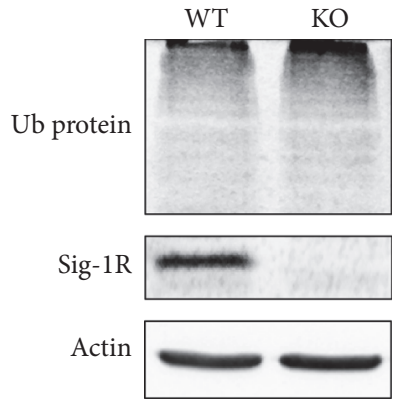

(a)

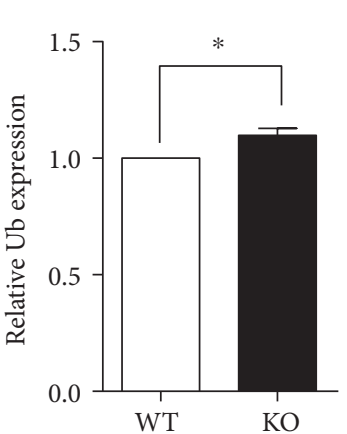

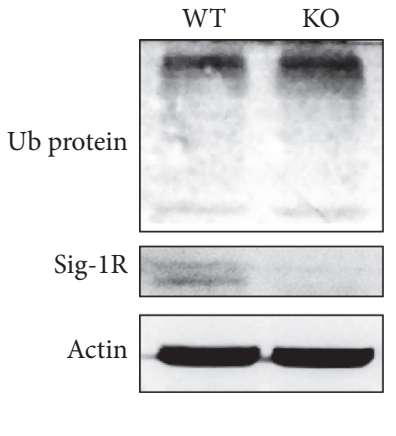

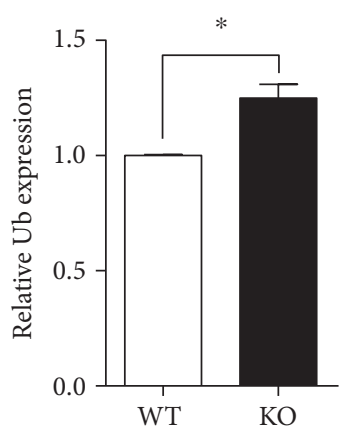

(b)

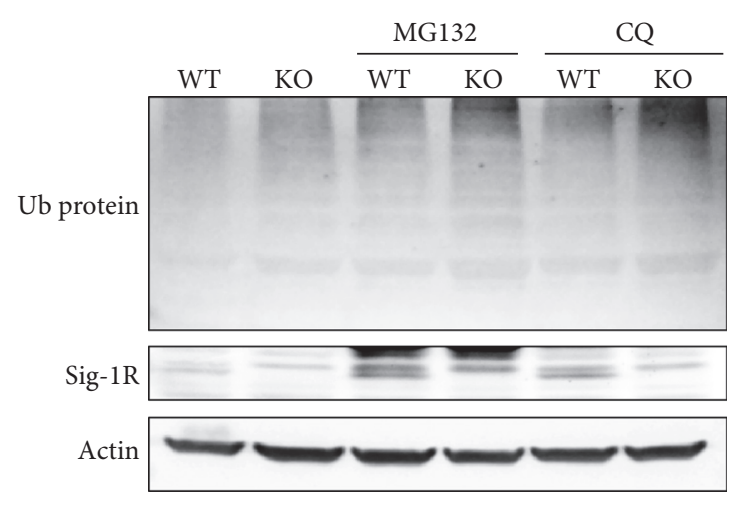

(c)

Figure 2: Sig-1R deficiency induced dysregulated protein degradation systems. The whole brains of (a) E18 fetuses $(N=3)$ or (b) $14 \sim 15$-month-old adult mice $(N=3)$ from Sig-1R WT or KO mice were collected and homogenized for immunoblotting. Sig-1R KO homogenates showed more ubiquitinated protein expression. Briefly, Ub protein or Ub in this figure. In the graph, ubiquitinated protein was normalized to the internal control. For the ubiquitinated protein expression in the fetus brain, it was further normalized to the respective size-matched WT fetus as 1 . The ubiquitinated protein expression level in the WT was normalized to 1 . Error bar indicates SEM. ${ }^{*} p<0.05, t$-test. (c) Mixed neuronal-glial cultures isolated from Sig-1R WT or KO E18 fetuses were treated with MG132 or CQ on DIV 11 for $24 \mathrm{~h}$. Lysates were collected for immunoblotting, and ubiquitinated protein levels were increased in Sig-1R KO neurons, especially after the inhibitor treatments.

conditions since DIV 0 (Figure 1(c)). Primary cultures from $\mathrm{KO}$ animals also tended to express fewer MAP2-positive neurons. These data further support the concept that Sig$1 \mathrm{R} \mathrm{KO}$ conditions favor astrocyte proliferation in mixed neuronal-glial cultures.

\subsection{Sig-1R KO Brains Have Dysregulated Protein Degradation} Systems. The pronounced astrocyte populations in Sig-1R $\mathrm{KO}$ mixed neuronal-glial cultures prompted us to examine ubiquitination levels in Sig-1R KO mice to tease out a potential mechanism that leads to astrocyte activation. Enhanced ubiquitination was observed in brains harvested from fetuses or adult mice (Figures 2(a) and 2(b)). To further define the pathological mechanism in Sig-1R KO neurons, mixed neuronal-glial cultures were treated with either the proteasome inhibitor MG132 or the autophagy flux inhibitor chloroquine (CQ). Pretreatment with MG132 or CQ further increased the levels of ubiquitinated proteins (Figure 2(c)) in Sig-1R KO primary cultures, suggesting that both protein degradation systems were dysregulated under Sig-1Rdeficient conditions and implicating that the Sig- $1 \mathrm{R}$ plays an important role in protein homeostasis. Sig-1R proteins also accumulated after the MG132 or CQ treatment. Proteins are either degraded through the classical ubiquitin-proteasome and the autophagy-lysosome pathways [44] although majority of proteins are degraded by the proteasome pathway. We next examined whether the Sig-1R is also degraded by the autophagy system.

3.3. Sig-1Rs and Autophagosomes Reside in Different Compartments. To test whether the Sig-1R is degraded by autophagy, Neuro-2a cells overexpressed with EYFP-tagged Sig-1Rs were treated with CQ to distinguish the degradative stage of autophagy. The Sig-1R EYFP showed the classical diffused ER pattern as previously described [11] but did not colocalize with LC3 or p62 in untreated cells (Figure 3). LC3 is recruited to the inner and outer membranes of autophagosomes where inner-bound LC3 is degraded after subsequent lysosome fusion [45]. Additionally, p62 is degraded by autophagy [46]. We speculated that the Sig-1R is degraded by proteasomes but not by autophagosomes since Sig-1R EYFP does not colocalize with LC3 (Figure 3(a)) or p62 puncta (Figure 3(b)) in the CQ-treated cells. The Sig-1R displayed a slightly different distribution pattern in treated cells, where the Sig-1R resides in different compartments from the isolated autophagosomes. This raised the possibility that Sig- 

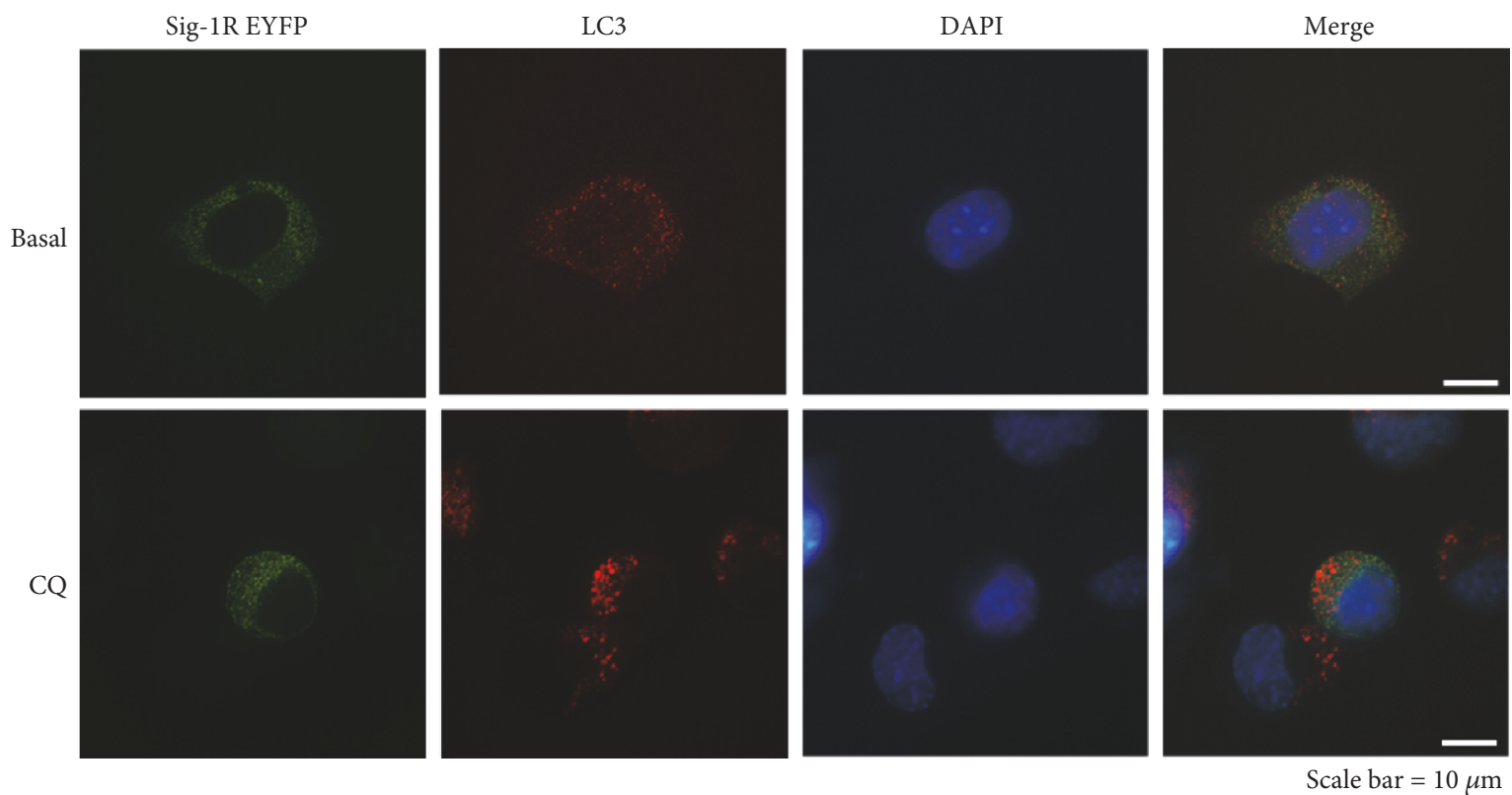

(a)
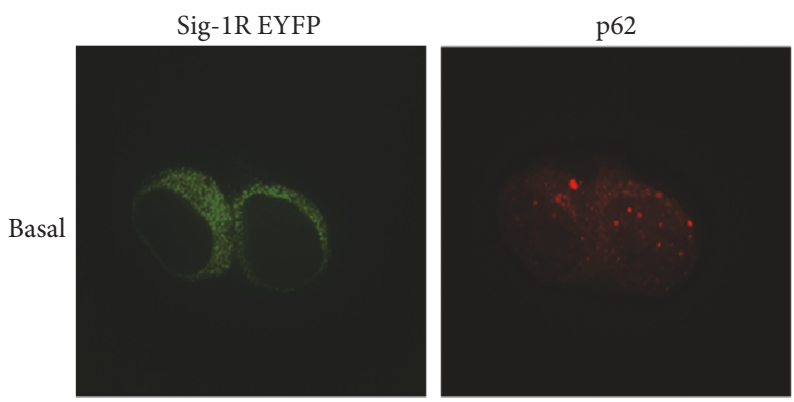

DAPI
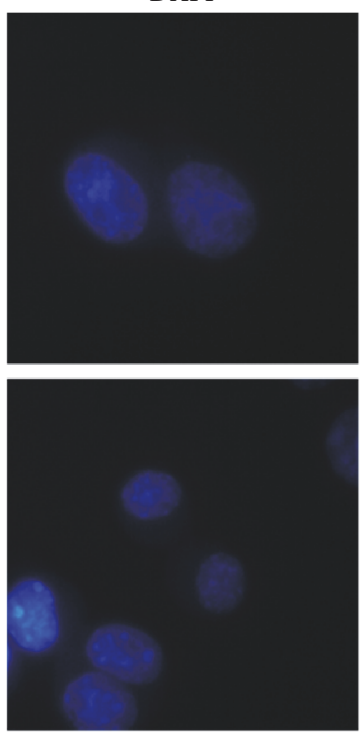

(b)

)

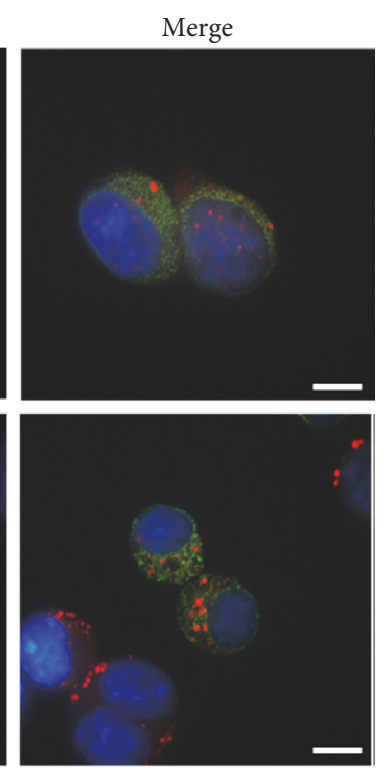

Scale bar $=10 \mu \mathrm{m}$
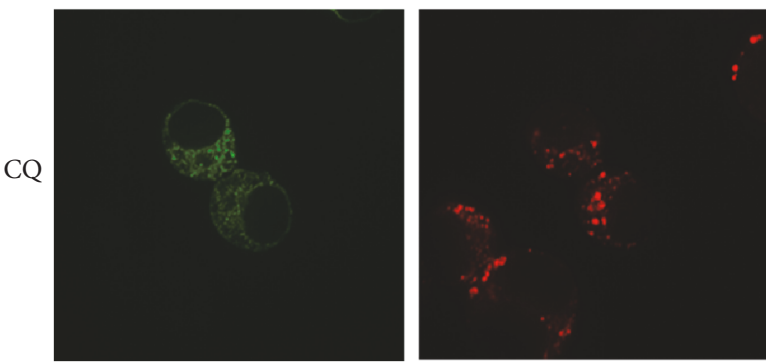

FIgURE 3: Sig-1R does not colocalize with autophagosomes. Neuro-2a cells were transiently transfected with Sig-1R EYFP for $24 \mathrm{~h}$ and followed by another $24 \mathrm{~h}$ of CQ treatments. Confocal images showed that the Sig-1Rs did not colocalize with (a) LC3, (b) p62, or the respective CQ-treated puncta.

1Rs do not aid in protein degradation through the latter stages of autophagy.

Because we know that silencing Sig-1Rs enhances free radical production [23] and because Sig-1Rs have been shown to be involved in Nrf2-mediated oxidative signaling $[36,38,39]$, we sought to examine the expression patterns of Nrf2 in Sig-1R KO neurons and how Nrf2 is regulated under oxidative stress conditions.

3.4. Nrf2 Signaling Is Enhanced in Sig-1R KO Neuronal-Glial Cultures upon Oxidative Stress. The characterization of the
Nrf2 proteins has been problematic in part due to the paucity of Nrf2 signals and in part due to the fact that the exact Nrf2 molecular weight observed by Western blotting analysis is still under debate [47]. We exogenously expressed Flagtagged Nrf2 in CHO cells and analyzed the molecular pattern using Flag antibodies as well as the Nrf2 antibody. In our system, Nrf2 showed a molecular weight of approximately $100 \mathrm{kDa}$ (Figure 4(a)). This result is in agreement with previous reports [47]. Notably, due to its quick turnover rate, Nrf2 was only detectable when cells were pretreated with proteasome inhibitors (Figures 4(a) and 4(b)); little or no Nrf2 


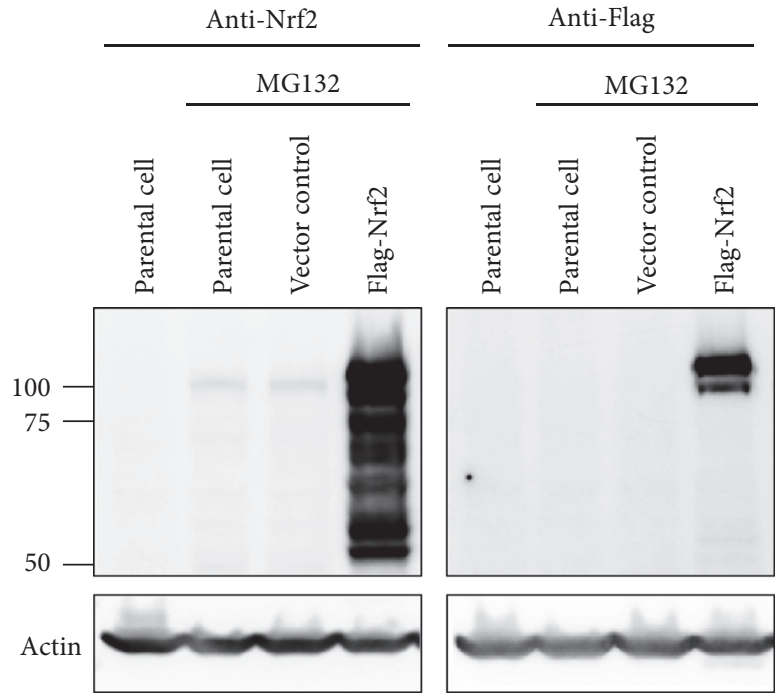

(a)

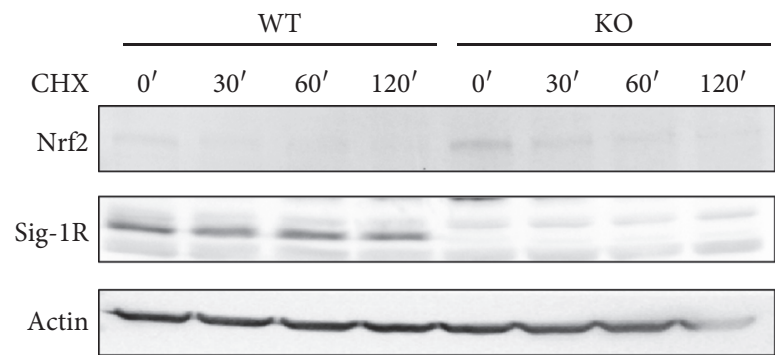

(c)

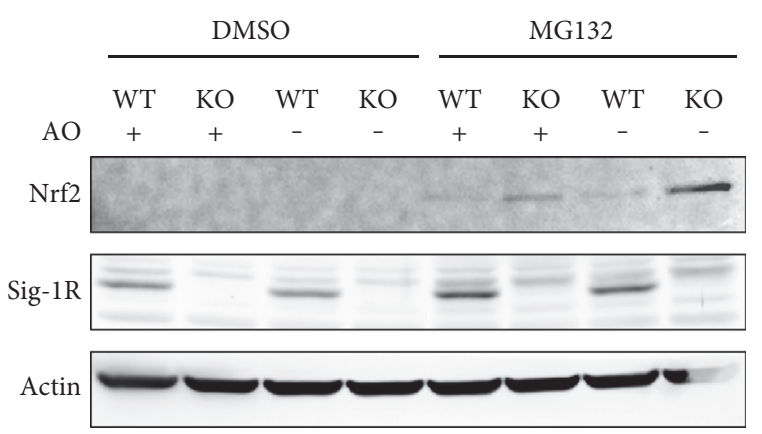

(b)
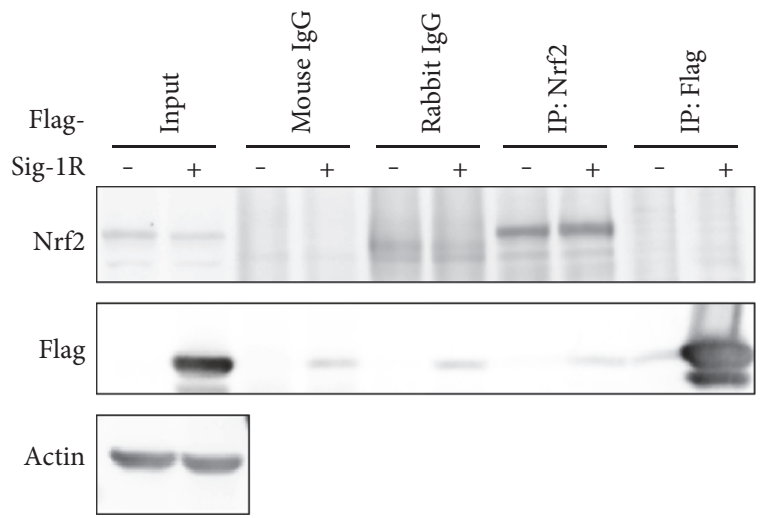

(d)

FiguRE 4: Sig-1R KO neuronal-glial cultures have enhanced Nrf2 expression. (a) CHO cells were transiently transfected with Flag-Nrf2 for $48 \mathrm{~h}$ and then treated with or without MG132 for $2 \mathrm{~h}$. Cell lysates were harvested and immunoblotted with Nrf2 or Flag antibodies. Note that only cells pretreated with MG132 have detectable Nrf2 signals. (b) Mixed neuronal-glial cultures isolated from Sig-1R WT or KO E18 fetuses were treated with DMSO or MG132 on DIV 11 with or without AO supplements for $24 \mathrm{~h}$. Cell lysates were collected, and the immunoblot showed that Nrf2 was increased in the KO primary cultures, especially when the Sig-1R KO cells were incubated in the AOfree medium. (c) Mixed neuronal-glial cultures isolated from Sig-1R WT or KO E18 fetuses were pretreated with MG132 for $2 \mathrm{~h}$ on DIV 11 followed by treatment with CHX. Cell lysates were collected at the indicated time points. (d) CHO cells were transiently transfected with Flag-Sig-1R for $48 \mathrm{~h}$ and then treated with MG132 for $2 \mathrm{~h}$ followed by immunoprecipitation. Nrf2 does not interact with Sig-1Rs.

was detectable in the control DMSO-treated cells. Therefore, cells were pretreated with MG132 to amplify Nrf2 expression levels throughout all experiments. To determine the expression of Nrf2 in Sig-1R KO neuronal-glial cultures, cells were pretreated with MG132 in combination with different conditioned media (culture medium supplemented with or without antioxidants (AO)). Our results showed that Sig-1R KO primary cultures exhibited higher Nrf2 levels than control cells. Additionally, Nrf2 levels were further enhanced in cells cultured without AO supplementation (Figure 4(b)). To analyze the Nrf2 protein stability, cycloheximide (CHX) was added to the primary cultures and cell lysates were collected at the indicated time points. There were faint Nrf2 signals in the WT group as observed in Figure 4(b), while a more pronounced signal was detected in the $\mathrm{KO}$ group, and it gradually decreased/diminished with $2 \mathrm{~h}$ of CHX treatment
(Figure 4(c)). Since the half-life of Nrf2 is within $30 \mathrm{~min}$, our data are consistent with previous reports that Nrf2 has fast degradation rates [48]. We also used an immunoprecipitation assay to examine the association between the Sig-1R and Nrf2. We found that these two proteins do not physically interact (Figure 4(d)), indicating that the Sig-1R does not regulate $\mathrm{Nrf} 2$ via a direct interaction. These results indicate that Nrf2 signaling is enhanced in Sig-1R KO neuronal-glial cultures by an as yet unknown mechanism.

3.5. Nrf2 Signaling Is Enhanced in Sig-1R KO Astrocyte and Cell Lines. Glia cells, especially astrocytes, have been demonstrated to preferentially protect neurons from the oxidative insults in the Nrf2-depedent pathway [49]. To further investigate the Nrf2 signaling in astrocyte under Sig-1R KO conditions, we isolated primary astrocytes from the 1-day-old 


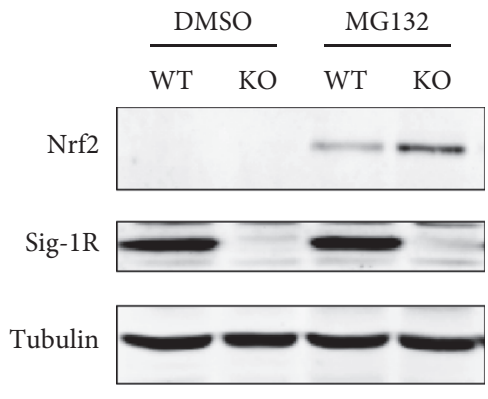

(a)
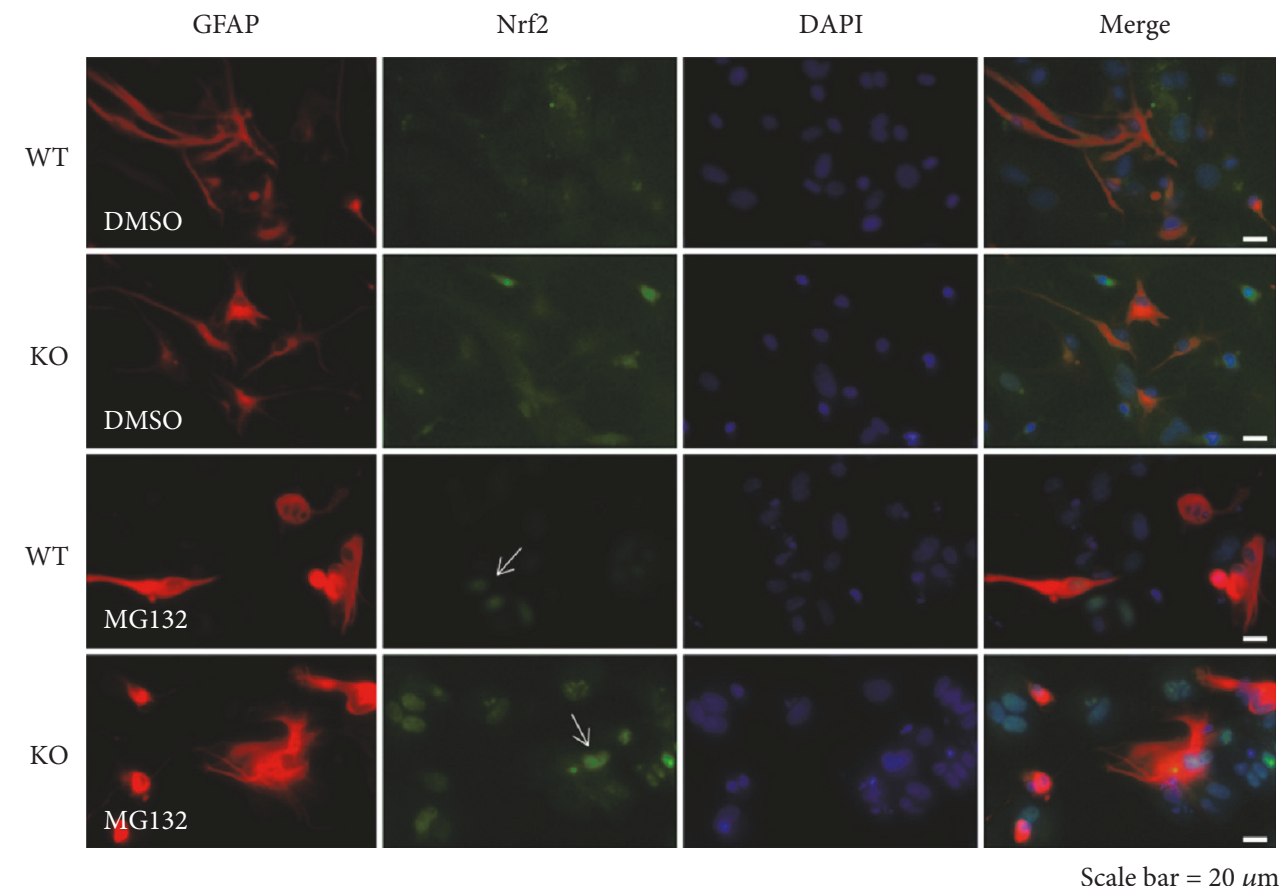

(b)

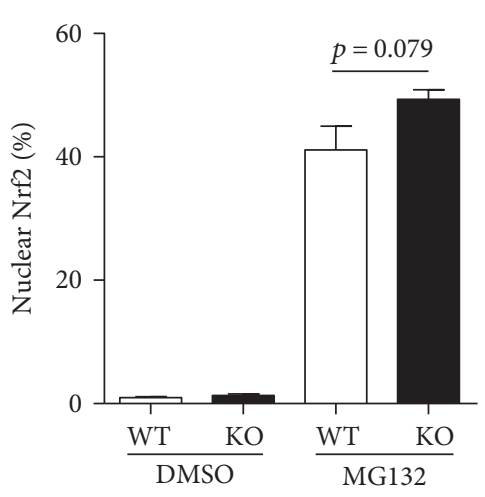

(c)
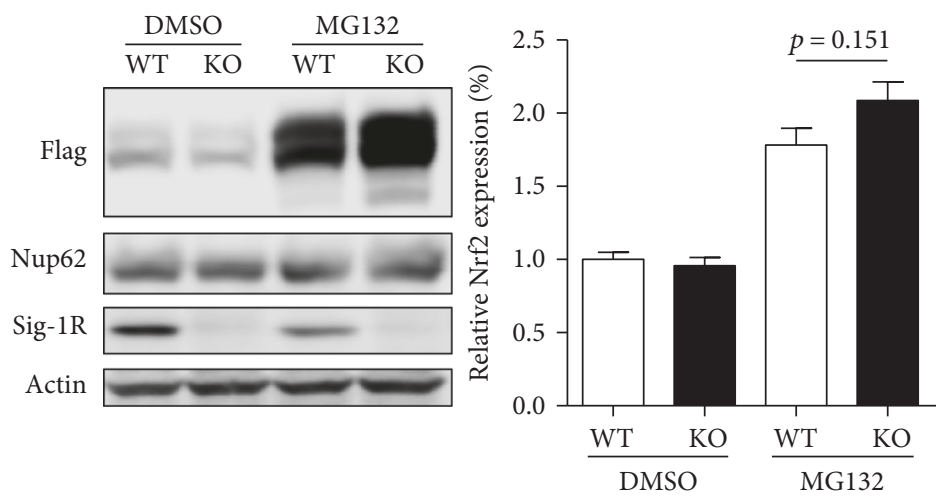

(d)

FIGURE 5: Nrf2 is enhanced in the Sig-1R KO cells under MG132 treatment. (a) Primary astrocytes isolated from Sig-1R WT or KO neonatal brains were treated with DMSO or MG132 for $24 \mathrm{~h}$. Cell lysates were collected, and the immunoblot showed that Nrf2 was increased in the KO cells compared with WT cells under MG132 treatment. (b) Isolated astrocyte cultures from Sig-1R WT or KO neonatal brains were treated with DMSO or MG132 for $24 \mathrm{~h}$ followed by staining with the Nrf2 and GFAP antibodies. MG132 induced the pronounced nuclear pattern of Nrf2 in WT and KO cultures, as shown by the arrow. (c) To obtain nuclear Nrf2 percentage, the number of nuclear Nrf2 positive cells was divided by the total cell numbers (as indicated by DAPI signal). The images were captured from 3-4 random fields per sample. (d) WT or Sig-1R KO HEK cells were transiently transfected with Flag-Nrf2 for $24 \mathrm{~h}$ and then treated with DMSO or MG132 for another $24 \mathrm{~h}$. Nuclear fractions were collected to determine the Nrf2 expression pattern. Nrf2 was normalized to the internal control. The Nrf2 expression level in the WT DMSO-treated group was normalized to 1. A trend of increased Nrf2 was observed in the Sig-1R KO cells. Statistical analysis was performed using $t$-test. Error bar indicates SEM. 
Normal state
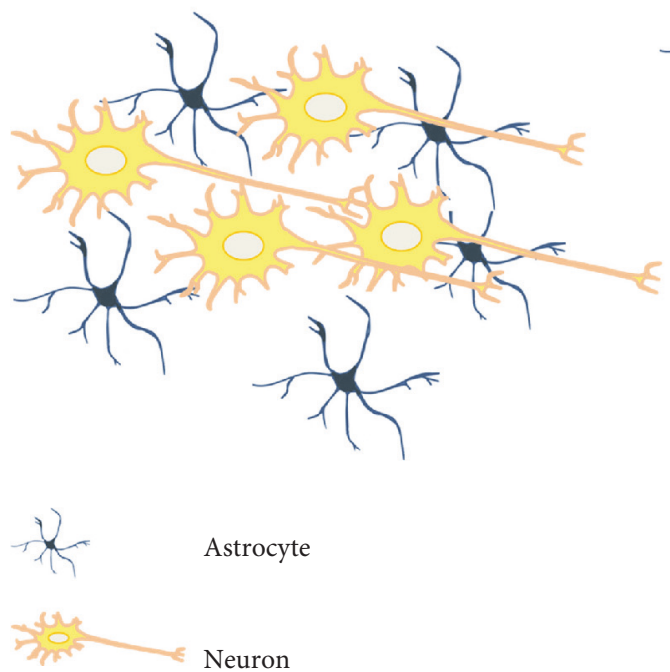

Sig-1R deficiency

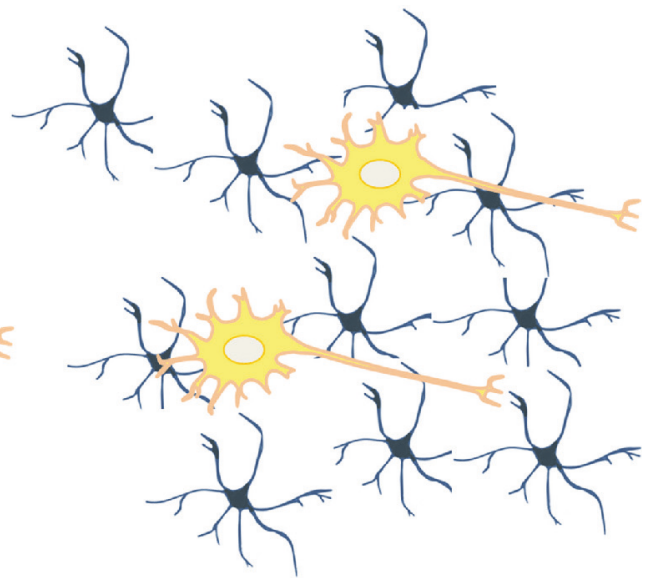

$$
\begin{aligned}
& \text { Astrogliosis } \uparrow \\
& \text { Neuronal loss } \uparrow \\
& \text { Ubiquitinated proteins } \uparrow \\
& \text { Nrf2 } \uparrow
\end{aligned}
$$

FIGURE 6: The proposed model for imbalanced interplay between neurons and astrocytes in Sig-1R deficiency pathologies. In the normal state, astrocytes maintain the homeostasis and functions of the neuronal cells. In the state of Sig-1R deficiency, an increased astrocyte population is seen at the early stage to cope with the stressful conditions, but this later becomes harmful to the neuronal cells and leads to neuronal loss. The ubiquitinated proteins are accumulated during this process and disrupt the proteostasis. The enhanced Nrf2 here may represent another compensatory mechanism via an as yet unknown mechanism.

neonatal mice. The Nrf2 expression level is enhanced in the Sig-1R KO astrocytes when compared with that in the WT astrocytes treated with MG132 (Figure 5(a)). Nrf2 is known to translocate into nucleus and activate ARE genes to combat the oxidative stress [40,41]. We examined the Nrf2 expression pattern by immunofluorescence. Nrf2 was detected in the nucleus from WT or KO cells (Figure 5(b)) in the presence of MG132 while a weak nuclear Nrf2 signal was observed in the control DMSO-treated groups. Although the nuclear expression of the Nrf2 did not reach the statistical significance ( $p=0.079)$ between the WT and Sig-1R KO astrocytes, there is a trend that the nuclear Nrf2 is increased in the Sig-1R KO astrocyte (Figure 5(c)), implying that astrocytes may need to activate Nrf2 in combating stress in Sig-1R KO condition. A different strategy was employed to further demonstrate that Nrf2 is increased in the nucleus. Exogenous Flag-Nrf2 was transfected to the WT or Sig-1R KO cells under MG132 treatments, followed by fractionation to separate nuclear protein. The results showed that Nrf2 exhibited abundantly in the nuclear fractionation and it dramatically accumulated after MG132 treatments in WT and KO cells where Nup62 was used as a nuclear marker (Figure 5(d)). Although the $p$ value did not show statistical significance $(p=0.151)$, an increased trend of Nrf2 in the nuclear fraction was observed from Sig-1R KO cells compared to the WT cells under proteasome inhibition. The enhanced Nrf2 pattern is consistent with the ICC results. Those results suggest that the Nrf2 is upregulated and accumulated in the nucleus in mixed neuronal-glial cultures and astrocytes as well as in the cell lines when the Sig-1R is depleted from those cells under stress.

\section{Discussion}

It is widely accepted that Sig-1R-mediated cellular functions are associated with the pathogenesis of many neurodegenerative disorders. In this study, we found that Sig-1R KO animals are prone to have imbalanced astrocyte populations, dysregulated metabolism, and enhanced Nrf2 signaling. These impaired cellular events may be a consequence of compensatory mechanisms caused by a Sig-1R deficiency (Figure 6). Astrocytes play critical roles in neuronal homeostasis including modulating glutamate uptake and recycling, regulating ion and energy production, and exerting oxidative stress protection [50]. Sig-1R KO animals exhibit a lower neurofilament density [10]. In addition, depletion of Sig1Rs creates an environment that is less favorable for neurons, causing neurons to become more vulnerable to cellular stress. Astrocytosis is observed in Sig-1R KO mixed neuronal-glial cultures (Figure 1), which could counterbalance a stressful microenvironment under the Sig-1Rdeficient condition. Gliosis has been found to occur in the affected regions of the CNS as means from adjacent tissue to limit the lesion size and to stimulate neuroprotection in regulation of CNS homeostasis. However, if gliosis goes out of control, adverse effects on neuroplasticity may occur [32].

Thus, the astrocytosis that is propagated in Sig- $1 \mathrm{R} \mathrm{KO}$ neuronal cultures may serve to protect neurons or on the other hand to limit neuronal growth under unfavorable conditions. Astrocytes maintain neuronal homeostasis by providing neurons with energy and nutrients and by maintaining a suitable microenvironment for neurons for example by defending neurons against antioxidant insult via the Nrf2- 
dependent pathway $[49,51]$. Another study has previously indicated that the Nrf2-induced gene upregulation signaling pathway was deficient in enriched neuronal cultures but was observed instead in astrocyte cultures, implicating the Nrf2 activation as well as the astrogliosis in Sig-1R-deficient cells [52]. Here, we demonstrated that Nrf2 is enhanced in both cell types, implying that neurons and astrocytes work cooperatively to survive under stressful conditions. Emerging evidence also shows that the Sig-1R mediates cellular functions via astrocyte activation. It has been demonstrated that Sig1 Rs can activate astrocyte functions through a positivefeedback mechanism under methamphetamine-stimulated conditions and the activation can be reversed by Sig-1R antagonist BD-1047 [35]. A recent study demonstrated that populations of activated GFAP-positive cells in Sig-1R KO mice were similar to those in WT mice in the substantia nigra. Interestingly, the study found that Sig-1R deficiency also led to reduction in MPTP-induced astrocyte activation [53]. Increased expression of GFAP in the cortex has been found in mice treated with cocaine for one week. Additionally, exposing astrocytes to cocaine resulted in rapid translocation of Sig-1Rs to the plasma membrane and activated downstream signaling pathways subsequently [54]. Chronic treatments of Sig-1R agonist PRE-084 decreased reactive astrocytosis in a motor neuron degeneration mouse model [21]. Another study also indicated that in Sig-1R KO impaired neurogenesis in the hippocampal dentate gyrus, suggesting that reduced survival without changes in the progenitor cell differentiation was seen in the absence of Sig-1R [55]. These studies indicate a critical role of Sig-1R in balancing astrocyte activation and neuron homeostasis. These become profound when the Sig-1R KO neurons are more vulnerable to cellular stress [10]. More experiments are warranted to tease out the brain regions that are more responsive to Sig-1R-mediated astrocytic regulation. Collectively, these data suggest that astrocytosis in Sig-1R KO brains is due to cellular responses that counterbalance neuronal damage via mechanisms yet to be discovered.

The increased accumulation of ubiquitinated proteins in Sig-1R KO neuronal-glial cultures becomes manifested when cells are treated with MG132 or CQ, suggesting that the metabolic regulation is compromised in Sig-1R deficiency when a stressful condition is imposed, such as ER stress or defective autophagy. The Sig-1R has been demonstrated as an ER sensor that facilitates interorganelle signaling to promote cell survival [9]; here, we also demonstrated that the Sig-1R plays an important role in proteostasis. An increase of Sig-1Rs was also observed after MG132 treatment (Figures 2(c) and 4(b)), we propose that Sig-1R may also be degraded through proteasome degradation system. Proteasome inhibition induced cell apoptosis, ROS production [56], and ER stress [57] and elevated the ER chaperones such as $\mathrm{BiP}[57,58]$. The Sig- $1 \mathrm{R}$ can attenuate the ER stress [59]; therefore, we speculate that the cellular stress caused by the MG132 may lead to partial induction of Sig-1R expressions. Additionally, we observed that Sig-1Rs are accumulated after the CQ treatment (Figure 2(c)) but did not colocalize with autophagosomes (Figure 3 ). We speculate that the upregulation of Sig-1Rs is induced due to the autophagy dysfunction. The accumulation of misfolded proteins affecting various cellsignaling systems is a shared hallmark in neurodegenerative diseases [60]. The Sig-1R participates in the degradation of proteins, such as UDP-galactose:ceramide galactosyltransferase [61], or intracellular inclusion, such as in Huntington's disease (HD) [62], via the ER-related degradation machinery. It is also interesting to note that the Sig-1R itself is accumulated and present in neuronal nuclear inclusions in various neurodegenerative diseases [63]. In vitro studies demonstrated that leptomycin B and thapsigarginsequestered Sig-1Rs in the nucleus together with p62 [63]. We found that the Sig-1R was not colocalized with p62 under CQ treatment (Figure 3(b)). The Sig-1R has been reported to regulate autophagy $[18,19]$. Sig-1Rs may interact with p62 differently in the proteasome and autophagy degradation systems. Studies have implied crosstalk between proteasome and autophagy degradation systems [44]. The protein folding and quality control system takes place in the ER [64], while autophagy organogenesis is suggested to originate at the MAM [65]. Given the specific location of Sig-1R in the ER at the MAM and facing the cytosol [66], it can be concluded that the Sig-1R is a multifunctional protein and plays important roles in both degradation systems.

Sig-1Rs exert neuroprotective actions to regulate free radical generation, oxidative stress, unfolded protein responses, immune responses, and survival pathways [67]. Earlier generations of homozygous Sig-1R KO mice had a lower survival rate after birth than the WT mice in our system. We previously demonstrated that silencing of Sig-1R results in accumulation of more ROS as compared to control $\mathrm{CHO}$ cells [68] or hippocampal neurons [23]. We believe that compensatory mechanisms, such as Nrf2 antioxidant signaling, might have been activated in the later breeds. The Sig-1R has been reported to modulate oxidative stress responses that are possibly involved in the regulation of neuroplasticity by Rac1 signaling [23], and treatment of bovine brain mitochondria with Sig-1R agonist- $(+)$ pentazocine led to ROS production [69]. ROS scavengers prevented Sig-1R siRNA induced downregulation of $\mathrm{Bcl}-2$, and silencing of Sig-1R also potentiated $\mathrm{H}_{2} \mathrm{O}_{2}$-induced cell death [70]. Reports also suggest that Sig-1R ligands such as PRE-084 can mitigate the effects of nitrosative and oxidative stress to proteins [71]. In a cellular model using Sig-1R KO Müller glia cells, Nrf2 expression and Nrf2-ARE-binding affinity were significantly decreased [36]. We speculate that these diverse results may be due to the employment of different cell types and experimental conditions. Transient silencing of Sig-1Rs in rat hippocampal neurons reduced the mRNA expression of HO- 1 and other ARE-element response genes (data not shown). The results were consistent with studies supporting the idea that the Sig-1R regulates oxidative stress via Nrf2. Therefore, we speculate that the Sig-1R may contribute to the acute-phase response of Nrf2. A recent study also redefined the role of $\mathrm{Nrf2}$, showing that $\mathrm{Nrf2}$ inactivation was required for proper neuronal development, and ectopic expression of Nrf2 caused defective neuron morphology and synaptogenesis, which results from the imbalance of the redox system [72]. Furthermore, Sig-1Rs not only protect cell against cellular stress [38] but also regulate lipid metabolism or transportation while Nrf2 is also involved in 
lipid homeostasis [73]. Sig-1R ligands, PRE-084 and pentazocine, alleviate $\mathrm{A} \beta$ peptide-induced lipid peroxidation in hippocampus [74] and retinal lipid oxidation [75], respectively. Lipid peroxidation is also increased in Nrf2compromised neurons [76]. However, the Sig-1R does not directly regulate or interact with Nrf2 (Figure 4(d)) in the resting condition, and the physical interaction between the multiple molecular partners [5] and Sig-1R may change when the cells are exposed to stressful circumstances. Our data indicated that other glia cells may also contribute to compensate the stressed condition as the nuclear Nrf2 pattern was also observed in GFAP-negative cells (Figure 5(b)). Furthermore, silencing of Sig-1R not only destabilized the lipid raft but also caused the defective autophagy [18], and dysregulation of autophagy has been shown to activate Nrf2 in a noncanonical pathway [77]. The enhanced Nrf2 signaling in Sig-1R KO neuronal-glial cultures, particularly astrocytes, may be a compensatory effect which promotes neuron survival and mitigates oxidative stress conditions.

It is possible that certain brain areas are more vulnerable to Sig-1R-deficiency-induced astrocyte activity. There may also be region-specific variations in astrocytic functions in response to stimulations. Sig-1R ligands have neuroprotective effects in ALS, AD, HD, PD, retinal degeneration, stroke, and methamphetamine-induced toxicity. These ligands may elicit neuroprotective effects via modulating ER homeostasis, balancing ROS, and both neuron and glial functions [78]. We demonstrated that Nrf2 is increased in the cell lines, the mixed neuronal-glia and astrocyte cultures from $\mathrm{KO}$ mice under stressed conditions. Our results suggest a potential compensatory mechanism caused by Sig-1R deficiency, addressing the important role of the Sig- $1 \mathrm{R}$ in protein quality control systems and in the balance between neurons and glial cells.

\section{Abbreviations}

$\begin{array}{ll}\text { AD: } & \text { Alzheimer's disease } \\ \text { ALS: } & \text { Amyotrophic lateral sclerosis } \\ \text { AO: } & \text { Antioxidants } \\ \text { ARE: } & \text { Antioxidant response element } \\ \text { Cdk5: } & \text { Cyclin-dependent kinase } 5 \\ \text { CHX: } & \text { Cycloheximide } \\ \text { CNS: } & \text { Central nervous system } \\ \text { CQ: } & \text { Chloroquine } \\ \text { ER: } & \text { Endoplasmic reticulum } \\ \text { GFAP: } & \text { Glial fibrillary acidic protein } \\ \text { HD: } & \text { Huntington's disease } \\ \text { IP3: } & \text { 1,4,5-Trisphosphate type 3 } \\ \text { MAM: } & \text { Mitochondrion-associated ER membrane } \\ \text { Nrf2: } & \text { Nuclear factor erythroid 2-related factor } 2 \\ \text { PD: } & \text { Parkinson's disease } \\ \text { ROS: } & \text { Reactive oxygen species } \\ \text { Sig-1R: } & \text { Sigma-1 receptor. }\end{array}$

\section{Conflicts of Interest}

The authors declare that they have no competing interests.

\section{Acknowledgments}

This work was supported by the Intramural Research Program of the National Institute on Drug Abuse, National Institutes of Health, and U.S. Department of Health and Human Services.

\section{References}

[1] T. Hayashi and M. Fujimoto, "Detergent-resistant microdomains determine the localization of sigma-1 receptors to the endoplasmic reticulum-mitochondria junction," Molecular Pharmacology, vol. 77, no. 4, pp. 517-528, 2010.

[2] S. Kourrich, T. Hayashi, J. Y. Chuang, S. Y. Tsai, T. P. Su, and A. Bonci, "Dynamic interaction between sigma-1 receptor and Kv1.2 shapes neuronal and behavioral responses to cocaine," Cell, vol. 152, no. 1-2, pp. 236-247, 2013.

[3] S. Y. Tsai, M. J. Pokrass, N. R. Klauer, N. E. D. Credico, and T. P. Su, "Sigma-1 receptor chaperones in neurodegenerative and psychiatric disorders," Expert Opinion on Therapeutic Targets, vol. 18, no. 12, pp. 1461-1476, 2014.

[4] R. R. Matsumoto, "Targeting sigma receptors: novel medication development for drug abuse and addiction," Expert Review of Clinical Pharmacology, vol. 2, no. 4, pp. 351-358, 2009.

[5] T. P. Su, T. C. Su, Y. Nakamura, and S. Y. Tsai, "The sigma-1 receptor as a pluripotent modulator in living systems," Trends in Pharmacological Sciences, vol. 37, no. 4, pp. 262-278, 2016.

[6] C. P. Palmer, R. Mahen, E. Schnell, M. B. Djamgoz, and E. Aydar, "Sigma-1 receptors bind cholesterol and remodel lipid rafts in breast cancer cell lines," Cancer Research, vol. 67, no. 23, pp. 11166-11175, 2007.

[7] S. Y. Tsai, T. Hayashi, T. Mori, and T. P. Su, "Sigma-1 receptor chaperones and diseases," Central Nervous System Agents in Medicinal Chemistry, vol. 9, no. 3, pp. 184-189, 2009.

[8] T. Hayashi and T. P. Su, "Sigma-1 receptor chaperones at the ER-mitochondrion interface regulate $\mathrm{Ca}^{2+}$ signaling and cell survival," Cell, vol. 131, no. 3, pp. 596-610, 2007.

[9] T. Mori, T. Hayashi, E. Hayashi, and T. P. Su, "Sigma-1 receptor chaperone at the ER-mitochondrion interface mediates the mitochondrion-ER-nucleus signaling for cellular survival," PLoS One, vol. 8, no. 10, article e76941, 2013.

[10] S. Y. Tsai, M. J. Pokrass, N. R. Klauer, H. Nohara, and T. P. Su, "Sigma-1 receptor regulates tau phosphorylation and axon extension by shaping p35 turnover via myristic acid," Proceedings of the National Academy of Sciences of the United States of America, vol. 112, no. 21, pp. 6742-6747, 2015.

[11] S. Y. Tsai, J. Y. Chuang, M. S. Tsai et al., "Sigma-1 receptor mediates cocaine-induced transcriptional regulation by recruiting chromatin-remodeling factors at the nuclear envelope," Proceedings of the National Academy of Sciences of the United States of America, vol. 112, no. 47, pp. E6562-E6570, 2015.

[12] A. van Waarde, A. A. Rybczynska, N. K. Ramakrishnan, K. Ishiwata, P. H. Elsinga, and R. A. Dierckx, "Potential applications for sigma receptor ligands in cancer diagnosis and therapy," Biochimica et Biophysica Acta, vol. 1848, no. 10, Part B, pp. 2703-2714, 2015.

[13] E. Aydar, P. Onganer, R. Perrett, M. B. Djamgoz, and C. P. Palmer, "The expression and functional characterization of sigma (sigma) 1 receptors in breast cancer cell lines," Cancer Letters, vol. 242, no. 2, pp. 245-257, 2006. 
[14] M. Gueguinou, D. Crottes, A. Chantome et al., "The SigmaR1 chaperone drives breast and colorectal cancer cell migration by tuning SK3-dependent Ca2+ homeostasis," Oncogene, vol. 36, no. 25, pp. 3640-3647, 2017.

[15] D. Das, L. Persaud, J. Dejoie et al., “Tumor necrosis factorrelated apoptosis-inducing ligand (TRAIL) activates caspases in human prostate cancer cells through sigma 1 receptor," Biochemical and Biophysical Research Communications, vol. 470, no. 2, pp. 319-323, 2016.

[16] M. Happy, J. Dejoie, C. K. Zajac et al., "Sigma 1 receptor antagonist potentiates the anti-cancer effect of p53 by regulating ER stress, ROS production, Bax levels, and caspase- 3 activation," Biochemical and Biophysical Research Communications, vol. 456, no. 2, pp. 683-688, 2015.

[17] T. Hayashi and T. P. Su, "Sigma-1 receptors ( $\sigma_{1}$ binding sites) form raft-like microdomains and target lipid droplets on the endoplasmic reticulum: roles in endoplasmic reticulum lipid compartmentalization and export," The Journal of Pharmacology and Experimental Therapeutics, vol. 306, no. 2, pp. 718725, 2003.

[18] J. T. Vollrath, A. Sechi, A. Dreser et al., "Loss of function of the ALS protein SigR1 leads to ER pathology associated with defective autophagy and lipid raft disturbances," Cell Death \& Disease, vol. 5, article e1290, 2014.

[19] L. Cao, M. P. Walker, N. K. Vaidya, M. Fu, S. Kumar, and A. Kumar, "Cocaine-mediated autophagy in astrocytes involves sigma 1 receptor, PI3K, mTOR, Atg5/7, Beclin-1 and induces type II programed cell death," Molecular Neurobiology, vol. 53, no. 7, pp. 4417-4430, 2016.

[20] Z. Wu, L. Li, L. T. Zheng, Z. Xu, L. Guo, and X. Zhen, “Allosteric modulation of sigma-1 receptors by SKF83959 inhibits microglia-mediated inflammation," Journal of Neurochemistry, vol. 134, no. 5, pp. 904-914, 2015.

[21] M. Peviani, E. Salvaneschi, L. Bontempi et al., "Neuroprotective effects of the sigma-1 receptor (S1R) agonist PRE084, in a mouse model of motor neuron disease not linked to SOD1 mutation," Neurobiology of Disease, vol. 62, pp. 218-232, 2014.

[22] K. T. Tchedre and T. Yorio, "Sigma-1 receptors protect RGC-5 cells from apoptosis by regulating intracellular calcium, Bax levels, and caspase-3 activation," Investigative Ophthalmology \& Visual Science, vol. 49, no. 6, pp. 2577-2588, 2008.

[23] S. Y. Tsai, T. Hayashi, B. K. Harvey et al., "Sigma-1 receptors regulate hippocampal dendritic spine formation via a free radical-sensitive mechanism involving Rac1xGTP pathway," Proceedings of the National Academy of Sciences of the United States of America, vol. 106, no. 52, pp. 22468-22473, 2009.

[24] A. Al-Saif, F. Al-Mohanna, and S. Bohlega, "A mutation in sigma-1 receptor causes juvenile amyotrophic lateral sclerosis," Annals of Neurology, vol. 70, no. 6, pp. 913-919, 2011.

[25] S. Watanabe, H. Ilieva, H. Tamada et al., "Mitochondria-associated membrane collapse is a common pathomechanism in SIGMAR1- and SOD1-linked ALS," EMBO Molecular Medicine, vol. 8, no. 12, pp. 1421-1437, 2016.

[26] M. Mishina, M. Ohyama, K. Ishii et al., "Low density of sigma1 receptors in early Alzheimer's disease," Annals of Nuclear Medicine, vol. 22, no. 3, pp. 151-156, 2008.

[27] N. Bernard-Marissal, J. J. Medard, H. Azzedine, and R. Chrast, "Dysfunction in endoplasmic reticulum-mitochondria crosstalk underlies SIGMAR1 loss of function mediated motor neuron degeneration," Brain, vol. 138, Part 4, pp. 875-890, 2015.
[28] T. A. Mavlyutov, M. L. Epstein, Y. I. Verbny et al., "Lack of sigma-1 receptor exacerbates ALS progression in mice," Neuroscience, vol. 240, pp. 129-134, 2013.

[29] V. Francardo, F. Bez, T. Wieloch, H. Nissbrandt, K. Ruscher, and M. A. Cenci, "Pharmacological stimulation of sigma-1 receptors has neurorestorative effects in experimental parkinsonism," Brain, vol. 137, Part 7, pp. 1998-2014, 2014.

[30] A. V. Molofsky, R. Krencik, E. M. Ullian et al., "Astrocytes and disease: a neurodevelopmental perspective," Genes \& Development, vol. 26, no. 9, pp. 891-907, 2012.

[31] Y. Chen and R. A. Swanson, "Astrocytes and brain injury," Journal of Cerebral Blood Flow and Metabolism, vol. 23, no. 2, pp. 137-149, 2003.

[32] M. Pekny, U. Wilhelmsson, and M. Pekna, "The dual role of astrocyte activation and reactive gliosis," Neuroscience Letters, vol. 565, pp. 30-38, 2014.

[33] N. J. Maragakis and J. D. Rothstein, "Mechanisms of disease: astrocytes in neurodegenerative disease," Nature Clinical Practice. Neurology, vol. 2, no. 12, pp. 679-689, 2006.

[34] J. Y. Moon, D. H. Roh, S. Y. Yoon et al., "Sigmal receptors activate astrocytes via p38 MAPK phosphorylation leading to the development of mechanical allodynia in a mouse model of neuropathic pain," British Journal of Pharmacology, vol. 171, no. 24, pp. 5881-5897, 2014.

[35] Y. Zhang, X. Lv, Y. Bai et al., "Involvement of sigma-1 receptor in astrocyte activation induced by methamphetamine via upregulation of its own expression," Journal of Neuroinflammation, vol. 12, p. 29, 2015.

[36] J. Wang, A. Shanmugam, S. Markand, E. Zorrilla, V. Ganapathy, and S. B. Smith, "Sigma 1 receptor regulates the oxidative stress response in primary retinal Müller glial cells via NRF2 signaling and system $\mathrm{x}_{\mathrm{c}}{ }^{-}$, the $\mathrm{Na}^{+}$-independent glutamate-cystine exchanger," Free Radical Biology and Medicine, vol. 86, pp. 25-36, 2015.

[37] B. Uttara, A. V. Singh, P. Zamboni, and R. T. Mahajan, "Oxidative stress and neurodegenerative diseases: a review of upstream and downstream antioxidant therapeutic options," Current Neuropharmacology, vol. 7, no. 1, pp. 65-74, 2009.

[38] A. Pal, D. Fontanilla, A. Gopalakrishnan, Y. K. Chae, J. L. Markley, and A. E. Ruoho, "The sigma-1 receptor protects against cellular oxidative stress and activates antioxidant response elements," European Journal of Pharmacology, vol. 682, no. 1-3, pp. 12-20, 2012.

[39] S. Y. Tsai, R. K. Rothman, and T. P. Su, "Insights into the sigma-1 receptor chaperone's cellular functions: a microarray report," Synapse, vol. 66, no. 1, pp. 42-51, 2012.

[40] J. A. Johnson, D. A. Johnson, A. D. Kraft et al., "The Nrf2-ARE pathway: an indicator and modulator of oxidative stress in neurodegeneration," Annals of the New York Academy of Sciences, vol. 1147, pp. 61-69, 2008.

[41] M. J. Calkins, D. A. Johnson, J. A. Townsend et al., "The Nrf2/ARE pathway as a potential therapeutic target in neurodegenerative disease," Antioxidants \& Redox Signaling, vol. 11, no. 3, pp. 497-508, 2009.

[42] N. Morioka, H. Abe, R. Araki et al., "A beta 1/2 adrenergic receptor-sensitive intracellular signaling pathway modulates CCL2 production in cultured spinal astrocytes," Journal of Cellular Physiology, vol. 229, no. 3, pp. 323-332, 2014.

[43] N. D. Camp, R. G. James, D. W. Dawson et al., "Wilms tumor gene on X chromosome (WTX) inhibits degradation of NRF2 protein through competitive binding to KEAP1 
protein," The Journal of Biological Chemistry, vol. 287, no. 9, pp. 6539-6550, 2012.

[44] V. I. Korolchuk, F. M. Menzies, and D. C. Rubinsztein, "Mechanisms of cross-talk between the ubiquitin-proteasome and autophagy-lysosome systems," FEBS Letters, vol. 584, no. 7, pp. 1393-1398, 2010.

[45] L. Wang, M. Chen, J. Yang, and Z. Zhang, "LC3 fluorescent puncta in autophagosomes or in protein aggregates can be distinguished by FRAP analysis in living cells," Autophagy, vol. 9, no. 5, pp. 756-769, 2013.

[46] G. Bjorkoy, T. Lamark, S. Pankiv, A. Overvatn, A. Brech, and T. Johansen, "Monitoring autophagic degradation of p62/ SQSTM1," Methods in Enzymology, vol. 452, pp. 181-197, 2009.

[47] A. Lau, W. Tian, S. A. Whitman, and D. D. Zhang, "The predicted molecular weight of Nrf2: it is what it is not," Antioxidants \& Redox Signaling, vol. 18, no. 1, pp. 91-93, 2013.

[48] H. K. Bryan, A. Olayanju, C. E. Goldring, and B. K. Park, “The Nrf2 cell defence pathway: Keap1-dependent and -independent mechanisms of regulation," Biochemical Pharmacology, vol. 85, no. 6, pp. 705-717, 2013.

[49] A. D. Kraft, D. A. Johnson, and J. A. Johnson, "Nuclear factor E2-related factor 2-dependent antioxidant response element activation by tert-butylhydroquinone and sulforaphane occurring preferentially in astrocytes conditions neurons against oxidative insult," The Journal of Neuroscience, vol. 24, no. 5, pp. 1101-1112, 2004.

[50] M. Belanger and P. J. Magistretti, "The role of astroglia in neuroprotection," Dialogues in Clinical Neuroscience, vol. 11, no. 3, pp. 281-295, 2009.

[51] P. S. Baxter and G. E. Hardingham, "Adaptive regulation of the brain's antioxidant defences by neurons and astrocytes," Free Radical Biology and Medicine, vol. 100, pp. 147-152, 2016.

[52] K. F. Bell, B. Al-Mubarak, J. H. Fowler et al., "Mild oxidative stress activates Nrf2 in astrocytes, which contributes to neuroprotective ischemic preconditioning," Proceedings of the National Academy of Sciences of the United States of America, vol. 108, no. 1, pp. E1-E2, 2011, author reply E3-4.

[53] J. Hong, S. Sha, L. Zhou, C. Wang, J. Yin, and L. Chen, "Sigma1 receptor deficiency reduces MPTP-induced parkinsonism and death of dopaminergic neurons," Cell Death \& Disease, vol. 6, article e1832, 2015.

[54] L. Yang, H. Yao, X. Chen, Y. Cai, S. Callen, and S. Buch, "Role of sigma receptor in cocaine-mediated induction of glial fibrillary acidic protein: implications for HAND," Molecular Neurobiology, vol. 53, no. 2, pp. 1329-1342, 2016.

[55] S. Sha, W. J. Qu, L. Li, Z. H. Lu, L. Chen, and W. F. Yu, "Sigma-1 receptor knockout impairs neurogenesis in dentate gyrus of adult hippocampus via down-regulation of NMDA receptors," CNS Neuroscience \& Therapeutics, vol. 19, no. 9, pp. 705-713, 2013.

[56] Y. H. Han, H. J. Moon, B. R. You, and W. H. Park, “The effect of MG132, a proteasome inhibitor on HeLa cells in relation to cell growth, reactive oxygen species and GSH," Oncology Reports, vol. 22, no. 1, pp. 215-221, 2009.

[57] H. S. Park, Y. Jun do, C. R. Han, H. J. Woo, and Y. H. Kim, "Proteasome inhibitor MG132-induced apoptosis via ER stress-mediated apoptotic pathway and its potentiation by protein tyrosine kinase p56lck in human Jurkat T cells," Biochemical Pharmacology, vol. 82, no. 9, pp. 1110-1125, 2011.
[58] K. T. Bush, A. L. Goldberg, and S. K. Nigam, "Proteasome inhibition leads to a heat-shock response, induction of endoplasmic reticulum chaperones, and thermotolerance," The Journal of Biological Chemistry, vol. 272, no. 14, pp. 90869092, 1997.

[59] T. Omi, H. Tanimukai, D. Kanayama et al., "Fluvoxamine alleviates ER stress via induction of sigma-1 receptor," Cell Death \& Disease, vol. 5, article e1332, 2014.

[60] D. Lindholm, H. Wootz, and L. Korhonen, "ER stress and neurodegenerative diseases," Cell Death and Differentiation, vol. 13, no. 3, pp. 385-392, 2006.

[61] T. Hayashi, E. Hayashi, M. Fujimoto, H. Sprong, and T. P. Su, "The lifetime of UDP-galactose:ceramide galactosyltransferase is controlled by a distinct endoplasmic reticulum-associated degradation (ERAD) regulated by sigma-1 receptor chaperones," The Journal of Biological Chemistry, vol. 287, no. 51, pp. 43156-43169, 2012.

[62] Y. Miki, K. Tanji, F. Mori, and K. Wakabayashi, "Sigma-1 receptor is involved in degradation of intranuclear inclusions in a cellular model of Huntington's disease," Neurobiology of Disease, vol. 74, pp. 25-31, 2015.

[63] Y. Miki, F. Mori, T. Kon et al., "Accumulation of the sigma-1 receptor is common to neuronal nuclear inclusions in various neurodegenerative diseases," Neuropathology, vol. 34, no. 2, pp. 148-158, 2014.

[64] K. Araki and K. Nagata, "Protein folding and quality control in the ER," Cold Spring Harbor Perspectives in Biology, vol. 3, no. 11, article a007526, 2011.

[65] M. Hamasaki, N. Furuta, A. Matsuda et al., "Autophagosomes form at ER-mitochondria contact sites," Nature, vol. 495, no. 7441, pp. 389-393, 2013.

[66] H. R. Schmidt, S. Zheng, E. Gurpinar, A. Koehl, A. Manglik, and A. C. Kruse, "Crystal structure of the human sigma receptor,” Nature, vol. 532, no. 7600, pp. 527-530, 2016.

[67] T. Hayashi, "Conversion of psychological stress into cellular stress response: roles of the sigma-1 receptor in the process," Psychiatry and Clinical Neurosciences, vol. 69, no. 4, pp. 179191, 2015.

[68] T. Mori, T. Hayashi, and T. P. Su, "Compromising sigma-1 receptors at the endoplasmic reticulum render cytotoxicity to physiologically relevant concentrations of dopamine in a nuclear factor-kappaB/Bcl-2-dependent mechanism: potential relevance to Parkinson's disease," The Journal of Pharmacology and Experimental Therapeutics, vol. 341, no. 3, pp. 663671, 2012.

[69] N. Natsvlishvili, N. Goguadze, E. Zhuravliova, and D. Mikeladze, "Sigma-1 receptor directly interacts with Rac1GTPase in the brain mitochondria," BMC Biochemistry, vol. 16, p. 11, 2015.

[70] J. Meunier and T. Hayashi, "Sigma-1 receptors regulate Bcl-2 expression by reactive oxygen species-dependent transcriptional regulation of nuclear factor kappaB," The Journal of Pharmacology and Experimental Therapeutics, vol. 332, no. 2, pp. 388-397, 2010.

[71] H. Dong, Y. Ma, Z. Ren et al., "Sigma-1 receptor modulates neuroinflammation after traumatic brain injury," Cellular and Molecular Neurobiology, vol. 36, no. 5, pp. 639-645, 2016.

[72] K. F. Bell, B. Al-Mubarak, M. A. Martel et al., "Neuronal development is promoted by weakened intrinsic antioxidant defences due to epigenetic repression of Nrf2," Nature Communications, vol. 6, p. 7066, 2015. 
[73] Y. Tanaka, L. M. Aleksunes, R. L. Yeager et al., "NF-E2-related factor 2 inhibits lipid accumulation and oxidative stress in mice fed a high-fat diet," The Journal of Pharmacology and Experimental Therapeutics, vol. 325, no. 2, pp. 655-664, 2008.

[74] J. Meunier, J. Ieni, and T. Maurice, "The anti-amnesic and neuroprotective effects of donepezil against amyloid beta 2535 peptide-induced toxicity in mice involve an interaction with the sigmal receptor," British Journal of Pharmacology, vol. 149, no. 8, pp. 998-1012, 2006.

[75] J. Wang, A. Saul, P. Roon, and S. B. Smith, "Activation of the molecular chaperone, sigma 1 receptor, preserves cone function in a murine model of inherited retinal degeneration," Proceedings of the National Academy of Sciences of the United States of America, vol. 113, no. 26, pp. E3764-E3772, 2016.

[76] M. Narasimhan, L. Mahimainathan, M. L. Rathinam, A. K. Riar, and G. I. Henderson, "Overexpression of Nrf2 protects cerebral cortical neurons from ethanol-induced apoptotic death," Molecular Pharmacology, vol. 80, no. 6, pp. 988-999, 2011.

[77] A. Lau, X. J. Wang, F. Zhao et al., “A noncanonical mechanism of Nrf2 activation by autophagy deficiency: direct interaction between Keap1 and p62," Molecular and Cellular Biology, vol. 30, no. 13, pp. 3275-3285, 2010.

[78] L. Nguyen, N. Kaushal, M. J. Robson, and R. R. Matsumoto, "Sigma receptors as potential therapeutic targets for neuroprotection," European Journal of Pharmacology, vol. 743, pp. 4247, 2014. 


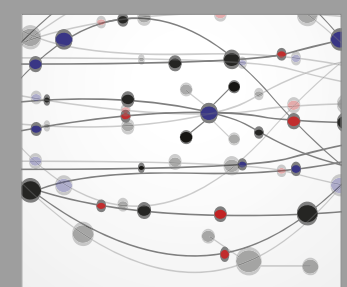

The Scientific World Journal
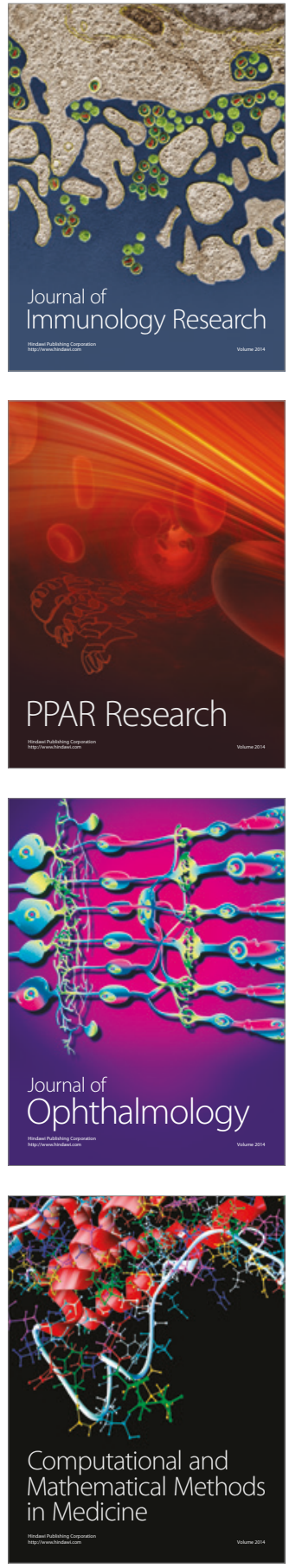

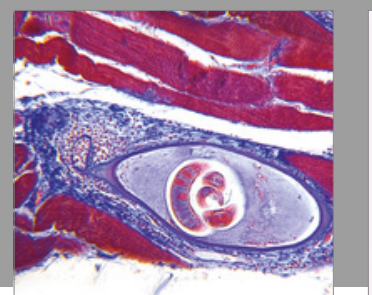

Gastroenterology Research and Practice
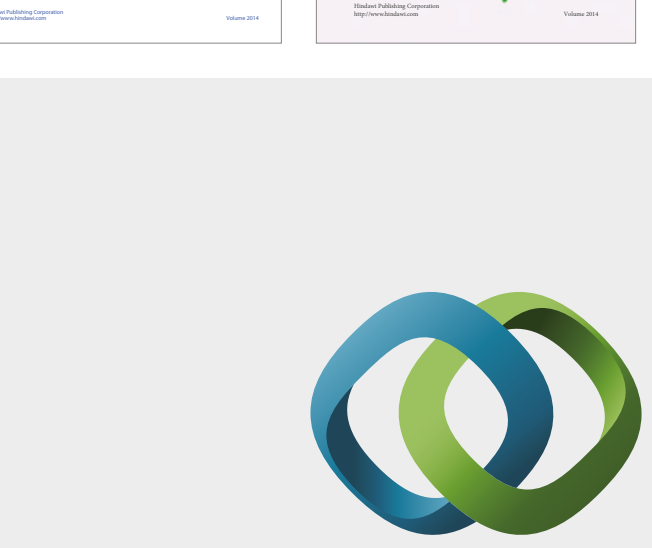

\section{Hindawi}

Submit your manuscripts at

https://www.hindawi.com
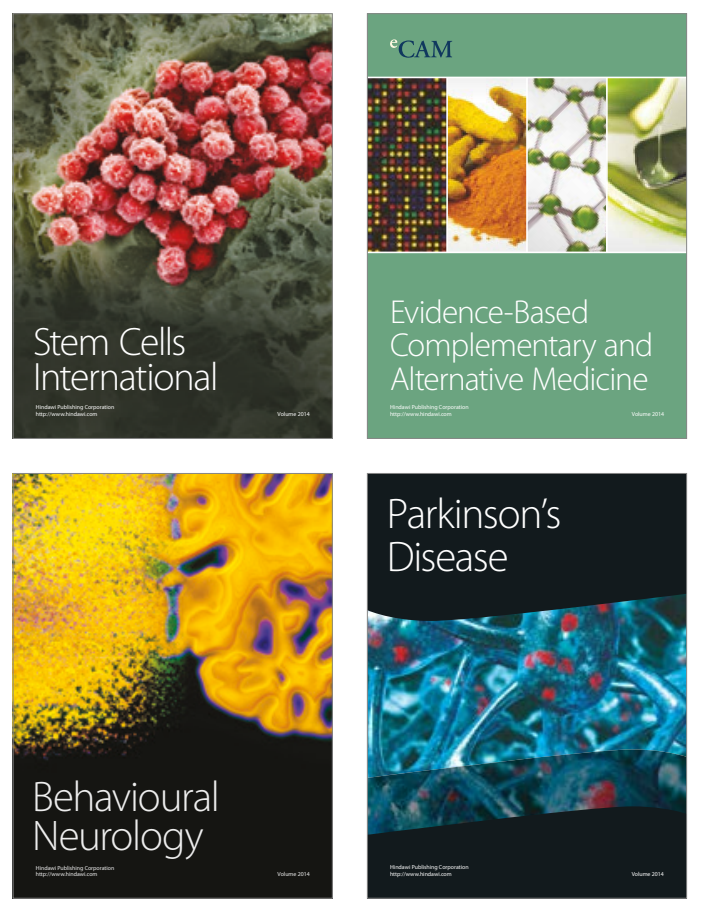
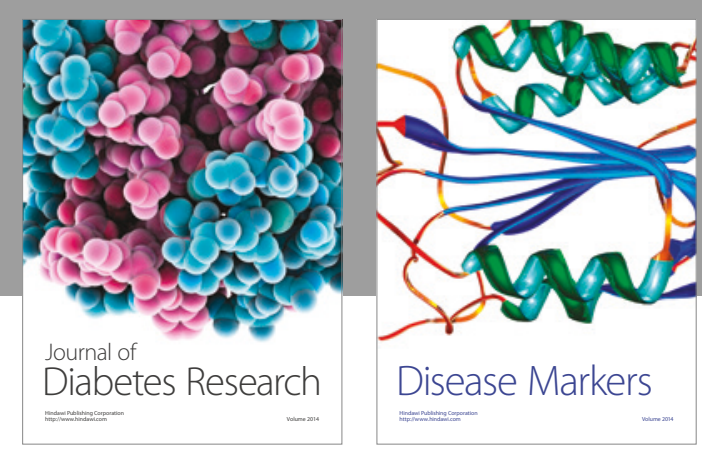

Disease Markers
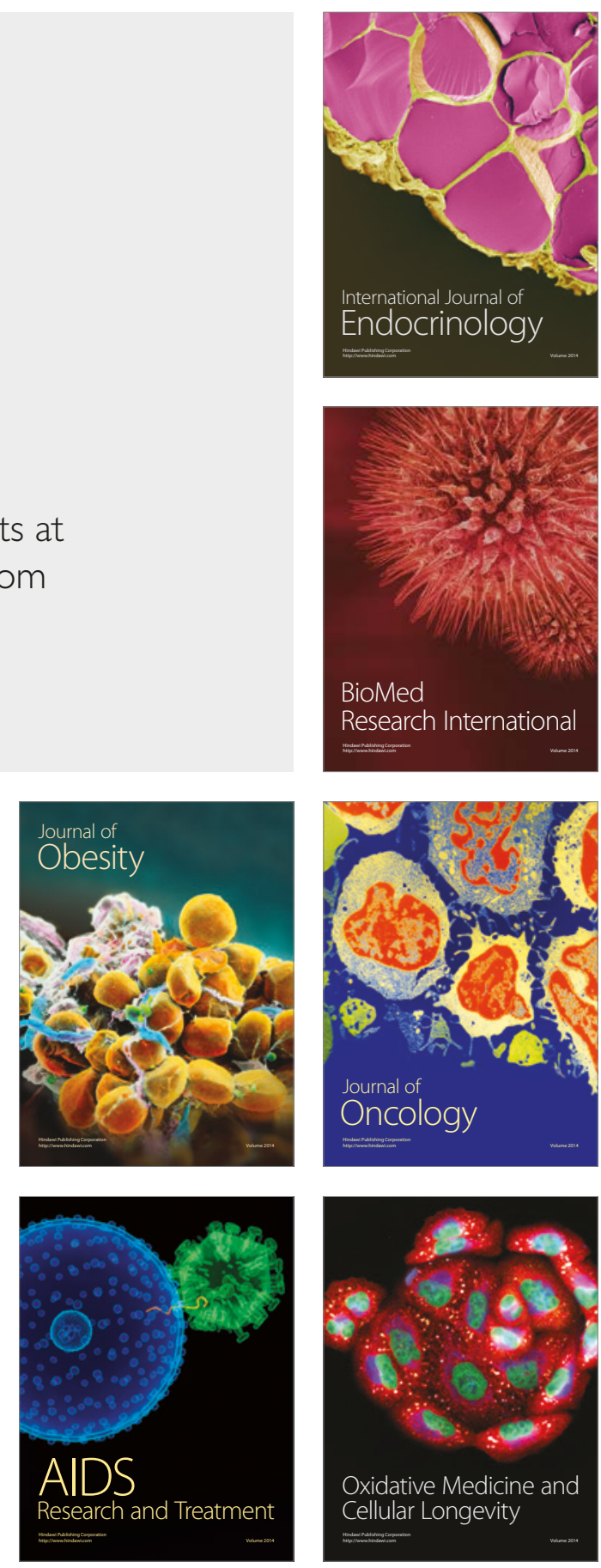